\title{
GERENCIAMENTO DE PROCESSOS DE NEGÓCIO E WORKFLOW: ESTADO DA ARTE E DELINEAMENTO CONCEITUAL
}

Ana Carolina Greef ${ }^{1}$

\begin{abstract}
RESUMO
Gerenciar processos exige dos atores no contexto de negócios a compreensão de conceitos, modelos e ferramentas apropriados para tal função. O Business Process Management (BPM) provê aos gestores desses ambientes a possibilidade de integrar pessoas e tecnologias para operacionalizar processos e alcançar objetivos organizacionais. O BPM, tratado como conjunto de metodologias e ferramentas é originário de outros mecanismos de gestão como o workflow. A similaridade de modelos e aplicações, bem como a relação histórica entre fluxos de trabalho e processos, levaram ao seu tratamento como sinônimos por parte de conteúdos da literatura e de ambientes gerenciados por meio desses modelos. Essa forma de tratamento dificulta a adoção correta de uma ou outra denominação para mecanismos e ferramentas de gestão organizacional, além de comprometer processos investigativos no contexto científico. Definições, componentes, aplicações e objetivos de BPM e workflow foram analisados por meio de investigação na literatura pertinente, nacional e internacional, publicada entre 2000 e 2012. Especificidades e complementaridades entre ambos foram levantadas, visando caracterizar cada qual e delimitar sua sobreposição conceitual, culminando em quadros de análise que permitem a visualização dos limites entre ambos. É demonstrada, enfim, uma proposta de delineamento conceitual dos temas investigados, contemplando suas características individuais e conjuntas, para viabilizar a correta utilização de cada termo nos contextos acadêmico e técnico.
\end{abstract}

Palavras-chave: Gerenciamento de Processos de Negócio. Workflow. Ciclo de vida de processos de negócio.

${ }^{1}$ Mestre, e-mail: ac.greef@gmail.com 


\section{INTRODUÇÃO}

Em 2002, consolidou-se a escola do Gerenciamento de Processos de Negócio ou Business Process Management (BPM), quando da publicação da obra BPM: thethirdwave por Smith e Fingar. Essa contribuição ocorreu em momento oportuno de discussão do potencial deste conceito em contribuir para a estruturação, a realização, a mensuração e a gestão de processos, em resposta à ausência de modelos robustos para gerenciar processos nos ambientes de negócio. Tal função era, até então, exercida por mecanismos como gestão de recursos, gestão da qualidade, engenharia de processos e (gestão de) fluxos de trabalho.

Nesse período, a administração do trabalho com foco em processos foi influenciada pela diversidade e evolução de conceitos e, principalmente, de tecnologias, o que tornou o BPM cada vez mais objeto de estudo nas áreas de gestão e de tecnologia (WESKE, 2007). O desenvolvimento paulatino da discussão do conceito e aplicações do BPM principalmente na área da administração, orientou a evolução de tais estudos, passando do foco no conceito propriamente dito para abordagens como ciclo de vida de processos, qualidade do trabalho, e cultura organizacional. O vínculo histórico do BPM com outras ferramentas gerenciais aplicadas nos ambientes organizacionais, entretanto, fez com que cada domínio desenvolvesse interpretações e formas particulares para sua inserção nos negócios.

A existência nos ambientes organizacionais de ferramentas de Gestão da Qualidade, de Reengenharia de Processos, de Enterprise Resource Planning (ERP), de Customer Relationship Management (CRM), e especialmente, de workflow, submeteu a inserção do BPM nesses contextos ao desafio de penetrar o conjunto de diversos mecanismos de gestão e tecnologias já inseridos nas organizações. Uma forma de minimizar a "juventude" do conceito de BPM frente a essa multiplicidade de mecanismos existentes nos referidos ambientes foi classificá-lo como evolução do workflow, já popularizado e operacionalizado nos negócios por sistemas específicos.

A proximidade de origens, funções e aplicações do BPM e do workflow, fez então com que ambos fossem tratados como similares e, frequentemente, como sinônimos, nos ambientes produtivos. Para os defensores do BPM, essa se constituiu uma oportunidade de inserção desse recurso no mercado, ao passo que representou uma ameaça para os desenvolvedores do workflow em seu conceito original. Estes últimos, especialmente no que se refere ao desenvolvimento de sistemas, passaram então a simplesmente modificar nomenclaturas e estilos de seus conceitos e produtos, para que parecessem soluções de BPM e, assim, pudessem permanecer no mercado.

Esse cenário contrariou a proposta da gestão de processos em organizar ambientes de trabalho, ao passo que, frequentemente, passou somente a somar um método e ferramenta novos a espaços já "lotados" de inúmeros outros recursos, desafiando a função gerencial sem, contudo, simplificá-la. Nesse ínterim, o conceito de Gerenciamento de Processos de Negócio passou a ser estritamente relacionado ao conceito de workflow 
no ambiente de negócios, o que não favoreceu seu desenvolvimento dissociado de sua relação histórica com os fluxos de trabalho.

Embora o ambiente acadêmico tivesse a oportunidade e mesmo a capacidade de definir e distinguir tais conceitos acompanhou a diversidade de aplicações e de concepções do BPM nas áreas de Administração, Engenharias, Produção e Qualidade, gerando abordagens diversas de definição de objetivos, componentes, funções e, inclusive relacionadas ao workflow. Em alguns casos, este último é apontado ora componente do BPM, ora o próprio, ora como seu antecessor histórico, o que dificulta a identificação de uma direção e definição conceitual única, que elimine sobreposições entre ambas as denominações.

Acredita-se, igualmente, que haja limites para definir cada um desses conceitos, fato que gera questionamentos como: que complementaridades existem entre o BPM e o workflow, que levam ao seu tratamento como elementos similares e até sobrepostos? Existem diferenças entre ambos os conceitos, aplicáveis a um esclarecimento dos limites teóricos que os envolvem visando o correto uso de uma ou de outra denominação?

Essas questões remetem ao estado da arte do Gerenciamento de Processos de Negócio e do workflow, cuja investigação é propósito deste artigo, que se propõe a contribuir para a formalização da distinção entre os conceitos investigados, e para o desenvolvimento dos métodos, técnicas e conceitos de gestão de processos considerando suas particularidades distintivas de suas áreas de base.

\section{PROCESSOS DE NEGÓCIO E FLUXOS DE TRABALHO}

A revisão de literatura da pesquisa contempla processos de negócio e fluxos de trabalho, histórico e definições, modelos conceituais e sistemas.

Os atores do ambiente de negócios realizam ações gerenciais sintetizadas na definição de suas estratégias, acompanhamento de indicadores e objetivos, monitoramento de desempenho, controle e correção de seu progresso. Os processos mostram-se ferramentas de operacionalização e de regulação dessas ações (MONTANINI et al., 2008) e sua gestão proporciona aos administradores estrutura para a melhoria de eficiência, a redução de custos e a qualidade de resultados, para satisfação de clientes (COSTA, 2009).

A importância estratégica dos processos enquanto elementos gerenciáveis que atribuem vantagem competitiva ao ambiente de negócios, é classificada por Santos (2003) como fonte de sinergia entre recursos organizacionais, derivando a necessidade de agilizar e aprimorar continuamente o trabalho (COSTA, 2009). Este último, integrado, suporta o controle, monitoramento e apoio à lógica do negócio, e traduz processos complexos em elementos gerenciáveis por conceitos, ferramentas e metodologias de administração de fluxos de trabalho. 
Os fluxos de trabalho provêm, à gestão de negócios, uma abordagem temporal, sequencial e funcional das atividades e informações inseridas no trabalho e compreendidas em processos (PÁDUA; BISPO, 2003). É devido a essa característica que o workflow representa, segundo o Workflow Management Coalition (1995), a passagem de trabalho entre atores, de acordo com regras e condições predefinidas, com objetivo de realizar atividades organizacionais.

A inserção de tecnologias para suporte à gestão de negócios transformou essa abordagem conceitual do workflow em uma abordagem estritamente ligada à tecnologia, fato reforçado por Weske, Van Der Aalst e Verbeek (2004) que acreditam que o foco do workflow é a realização de processos operacionais por meio de software. Tais sistemas, por sua vez, automatizam os fluxos de trabalho inserido nos processos de um negócio (DE SORDI; MARINHO, 2005).

\subsection{Aspectos conceituais e históricos}

Retomando a questão conceitual, a automação do trabalho provida pelo workflow ocorre devido à sua proposta de simplificação de atividades ou procedimentos do negócio em que está inserido, especialmente quanto ao fluxo de documentos entre unidades ou funções, que é agregando de eficiência (LAUDON; LAUDON, 2007). No contexto de comunicação em que esse fluxo de documentos ocorre, Verma (2009) ressalta que o workflow representa uma série de ações resultantes de eventos e atividades realizados por pessoas. Apesar do caráter operacional dos fluxos de trabalho atribuído por esses autores, Weske (2007) afirma que o workflow se trata de um conceito de gestão organizacional, que compreende a representação, em modelos, de processos de negócio, e a realização de forma flexível e adaptável desses processos. Costa (2009), de acordo com a visão de Weske (2007), afirma que esse gerenciamento de workflow tem estrita relação com áreas e atividades estratégicas das organizações. Sua visão é justificada pela conceituação do workflow como mecanismo de coordenação de atividades, associado por Georgakopoulos et al. (1995 apud COSTA, 2009) às ações de especificação, de reengenharia e de automação de processos de negócio.

A operacionalização dessas definições, passando do âmbito conceitual para o âmbito prático, exigiu a inserção dos fluxos de trabalho em ferramentas de tecnologia, que passaram então a representar o conceito em si. Esse fato é justificado pela visão de que o workflow ocorre necessariamente inserido em sistemas, de forma automática ou com algumas possibilidades de interação humana, para suportar os elementos automatizados dos processos e controlar atividades realizadas com base em modelos (WESKE, 2007). De forma menos sutil, Ko (2009) atribui ao workflow o papel de ferramenta ou conjunto de ferramentas de Tecnologia da Informação, que viabiliza o trâmite de documentos e a realização de transações de um negócio entre seus diversos componentes. Costa (2009), por sua vez, afirma que as dependências e regras dos fluxos de trabalho automatizados somente podem ser executadas por tecnologias. 
Tais aspectos revelam não somente a dissociação do workflow do trabalho coordenado e realizado por pessoas, mas também seu distanciamento do conceito de gestão do ambiente de trabalho, desviando o foco de uma administração holística do negócio em benefício da gestão dos elementos pontuais dos fluxos de trabalho, que nem sempre contemplam todos os componentes de uma organização.

No início do século XXI, a competição cada vez mais acirrada por clientes e resultados positivos passou a exigir das organizações a coordenação de atividades e de processos de trabalho, além da adaptabilidade a formatos de negócio que não aqueles já consolidados no mercado. O workflow já não mais seria suficiente para suprir tal necessidade na gestão organizacional, e novos mecanismos passaram a ser necessários, o que determinou a implementação do BPM (ENOKI, 2006).

Oportunamente, após a sistematização do conceito de BPM por Smith e Fingar, esse conceito passou a ser desenvolvido por estudiosos das áreas da administração e tecnologia, para que pudesse ser implementado nos ambientes organizacionais, sob a ótica da estruturação dos negócios em processos para atribuição de eficiência, redução de custos, obtenção de produtividade e agilidade ao desenvolvimento e à administração de processos.

Já Weske, Van Der Aalst e Verbeek (2004) definiram o BPM como o suporte a processos de negócio por meio da utilização de métodos, técnicas e software para promover o design, a execução, o controle e a análise de processos operacionais integrando pessoas, organizações, aplicações, documentos e outras fontes de informação. Esta última definição já atribui ao BPM tarefas específicas, distintas do papel assumido pelo workflow no ambiente de negócios.

Destaca-se, na definição proposta por Weske, Van Der Aalst e Verbeek (2004), o fato de que o BPM permite coordenar e adaptar o trabalho por meio de conhecimentos "ferramentais e organizacionais", respectivamente relativos às Tecnologias da Informação, e às teorias e metodologias de gerenciamento de processos, conforme Cruz (2006). A implementação dos domínios ferramental e organizacional oferece, como benefício aos negócios, segundo Miers (2006), o aprimoramento de desempenho subsidiado por uma cultura que envolve e integra pessoas, a forma como trabalham, tecnologias que utilizam e seus objetivos.

McCoy et al. (2007) apontam que esses objetivos são obtidos, além dos componentes já citados do BPM, por meio dos métodos, políticas, regras, métricas, práticas e ferramentas de Tecnologia da Informação, inseridos no conceito de Gerenciamento de Processos de Negócio. Já Montanini et al. (2008) acreditam que o aprimoramento citado por Miers (2006), bem como seus indicadores, variam conforme as características e processos de cada negócio, os quais devem ser automatizados, sendo essa uma das funções do BPM. Assim, o Gerenciamento de Processos de Negócio influencia comportamentos, táticas e tecnologias do ambiente em que está inserido (GARTNER GROUP, 2005 apud AMARAL et al., 2008). 
A influência mencionada pelo Garner Grupo interfere nos ambientes de negócio cujas culturas foram sendo estabelecidas ao longo do tempo, especialmente no que se refere à inserção contínua de ferramentas e mecanismos gerenciais aos quais as organizações se adaptaram a cada nova modalidade de gestão. A adequação dos negócios à execução eficiente e efetiva de processos depende da transição dessas estruturas organizacionais tradicionais para o que Costa (2009) chama de visão orientada a processos, que compreende "plataformas técnicas" para coordenação desses e para condução de seu ciclo de vida.

Um dos desafios impostos aos negócios nessa transição é a própria conceituação de processos e a sua definição no âmbito do negócio. Ko (2009) afirma que a conceituação tradicional de processos como "coleções de atividades" se deve à necessidade de sua compreensão como trabalho sistemático e ordenado ao longo do tempo. Essa definição, entretanto, pode reforçar similaridades estruturais entre o Gerenciamento de Processos de Negócio e a execução de fluxos de trabalho, ignorando atores envolvidos nos trabalhos sistemáticos, seus papéis e sua natureza colaborativa. O próprio Ko (2009) afirma que, nesse caso, pessoas, e sistemas constituem atores trabalhando em conjunto para alcançar objetivos de negócio e que precisam ser considerados na inserção do BPM nesse ambiente.

Essa inserção é facilitada por meio de modelos conceituais, modelos de referência e ciclos de vida, cuja aplicação está estritamente relacionada às tecnologias que operacionalizam os mesmos modelos.

\subsection{Modelos conceituais}

No caso do workflow, um modelo conceitual foi criado em 1993 para definir princípios universais a serem seguidos por softwares de fluxos de trabalho (VERMA, 2009). O BPM, por sua vez, não conta com modelo conceitual similar, visto que não é "regulamentado" por um único órgão, tampouco praticado de maneira padronizada em organizações (CRUZ, 2010). Esse fato dificultou a consolidação do BPM nos ambientes em que o modelo conceitual do workflow já era praticado.

Essa questão foi superada pela estruturação do ciclo de vida de processos de negócio e seu gerenciamento (Figura 1), que compreende etapas operacionalizadas simultaneamente por BPM e workflow, e diferencia ambos, conforme Weske (2007), por meio da função de avaliação de processos. 
Figura 1: Ciclo de vida de processos de negócio e papéis do BPM e do workflow

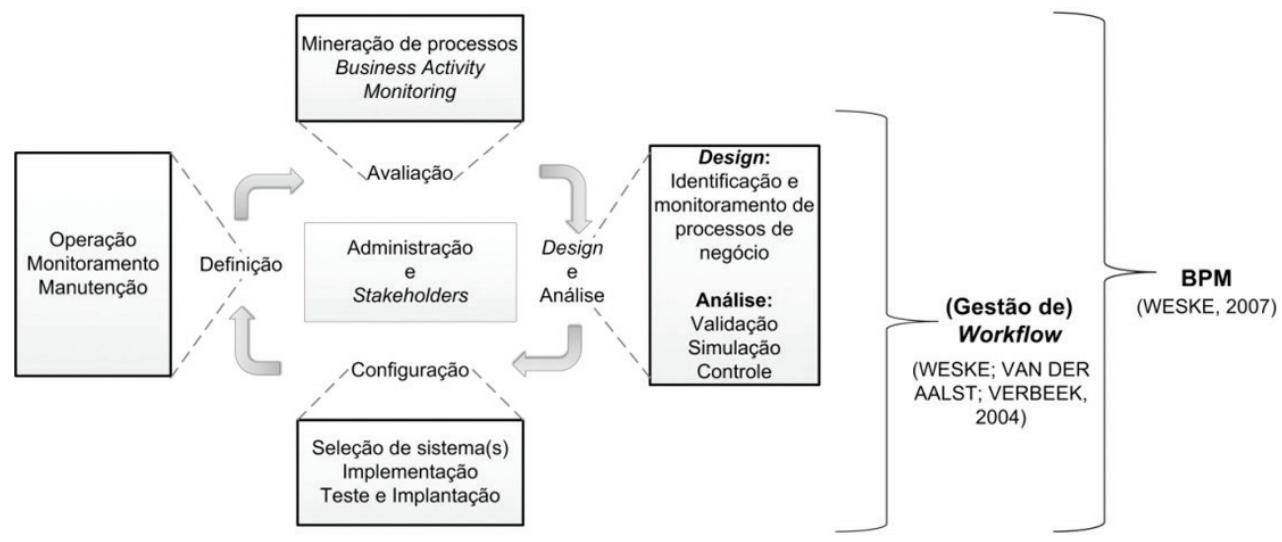

Fonte: Adaptado de Weske, Van Der Aalst e Verbeek (2004) e Weske (2007)

O ciclo ilustrado na Figura 1 contribui para uma primeira delimitação dos conceitos de BPM e workflow, permitindo ainda, entretanto, a sobreposição entre ambos no que tange às tarefas de design, configuração e definição de processos. Justamente essa sobreposição possibilitou aos desenvolvedores de sistemas de workflow fazer modificações tópicas nos produtos por eles desenvolvidos, aproximando-os do conceito de BPM sem necessariamente promover a reestruturação de toda a ideologia de base dessas ferramentas.

\subsection{Tecnologias de BPM e workflow}

A também oportuna ausência de delineamento de ambos os conceitos facilitou esse processo, em que se aproveitou a existência dos sistemas denominados Workflow Management Systems (WfMS) para realizar, por meio deles, o desenho, a integração, a automação e a gestão de processos (CARDOSO; BOSTROM; SHETH, 2004).

Segundo Reijers, Song e Jeong (2007), os WfMS disponibilizados na década de 2000 ofereceram mecanismos para distribuir o trabalho entre pessoas e tecnologias, habilitando a cooperação entre esses usuários na realização dos processos de negócio. Assim, esses Sistemas tiveram papel determinante no gerenciamento logístico de processos, agregando a eles agilidade e eficiência, enquanto o conceito e ferramentas de BPM ainda eram introduzidos no mercado e existia a oportunidade de manter ferramentas antigas para ocupar essas funções, para as quais nem sempre eram destinadas originalmente..

Frente a esse contexto, Costa (2009) afirma que o papel dos WfMS é, na verdade, de automatizar, gerenciar e executar fluxos de trabalho, tramitando tarefas e informações entre seus usuários por meio de regras e papéis, representações gráficas e modelos de workflow. As funções de gerenciar e realizar processos de negócio, com base em um 
design específico, é função dos chamados Business Process Management Systems (BPMS) (WESKE; VAN DER AALST; VERBEEK, 2004), criados especificamente para operacionalizar o BPM nas organizações e eliminar o uso indevido de WfMS na função de gerir processos.

Miers (2006), Leite e Rezende (2007) e Montanini et al. (2008) compartilham a visão de que os BPMS têm as funções de modelagem, execução, interoperabilidade, monitoramento e análise de processos de negócio, baseando-se em tecnologias já existentes nesse ambiente e integrando-as em uma única plataforma. Segundo Dubouloz (2004 apud OLIVEIRA et al., 2010) essas funções culminam no suporte ao ciclo de vida de processos de negócio como um todo, diferentemente dos WfMS, cujas funcionalidades oferecem suporte a algumas das etapas desse ciclo.

Nesse contexto, Cruz (2010) cita o Business Process Management Institute para classificar os BPMS como sistemas que promovem integração do negócio extrapolando o nível da tecnologia, uma vez que abrangem a interação dos stakeholders do negócio com sua execução propriamente dita. Este último aspecto, a princípio, diferencia os BPMS das demais tecnologias já inseridas na gestão dos ambientes organizacionais, o que, por conseguinte, motiva à implementação do BPM como estratégia de gerenciamento dos negócios, frente a outros modelos.

Essa inserção, apesar da proposta consolidada dos sistemas para operacionalização do BPM, com base no ciclo de vida do Gerenciamento de Processos de Negócio, também apresentou suas complexidades, pois processos organizacionais, em muitos casos, já eram gerenciados por outras ferramentas, inclusive WfMS, Nesse sentido, a distinção entre as funções destes últimos Sistemas e os BPMS também percorreu os desafios de delineamento de cada sistema e compreensão de seus papéis, idealmente distintos, na administração dos negócios.

Além disso, a literatura relaciona os WfMS e BPMS sob diversos aspectos: Cruz (2006) aponta que a administração do ciclo de vida do workflow é conceito genérico de base para os BPMS, inclusive por estar inserido nestes últimos Sistemas como uma de suas funcionalidades. Verma (2009), ainda afirma que os WfMS representam ferramentas de design de processos que viabilizam sua modelagem, e habilitam os BPMS a integrar pessoas e soluções de tecnologia. Ko, Lee e Lee (2009), por sua vez, acreditam que os WfMS têm funcionalidades para diagnóstico, design, configuração, avaliação e realização de processos, apesar de estas representarem etapas do ciclo de vida do BPM. Enfim, Costa (2009) considera os WfMS plataformas de coordenação de processos por meio do gerenciamento dos fluxos de trabalho, inseridas nos BPMS, ao passo que, para Cruz (2010), os WfMS constituem funcionalidades dos BPMS, por meio das quais processos são automatizados viabilizando o monitoramento, a mensuração e a avaliação contínuos de seu desempenho (CRUZ, 2010).

Essas, entre tantas vertentes de caracterização do workflow e do BPM quanto a conceitos e definições, tarefas, ciclos de vida e tecnologias, constituem os construtos deste estudo e servem ao objetivo de pesquisa de traçar o estado da arte e um delineamento conceitual 
de ambos os temas investigados. Apresentado o panorama teórico que fundamenta a pesquisa, a seção seguinte apresenta os procedimentos metodológicos utilizados em relação ao objetivo e aos questionamentos propostos para o estudo.

\section{PROCEDIMENTOS METODOLÓGICOS}

Para responder ao objetivo de "traçar o delineamento entre os conceitos BPM e de workflow por meio da síntese da literatura a respeito de ambos", identificando especificidades e complementaridades de conceitos e tecnologias de BPM e workflow, utilizou-se de pesquisa bibliográfica e de procedimentos similares aos utilizados por Rautenberg, Todesco e Steial (2010) e Moreno e Santos (2012) em pesquisas de escopo similar a esta.

O método hipotético-dedutivo baseou essa pesquisa, tendo sido estabelecido como hipótese para o estudo, o entendimento de que existem aspectos distintivos entre 0 BPM e o workflow que, no entanto, foram pouco destacados na literatura até o presente momento, devido à diversidade de vertentes de pesquisa adotadas por obras e autores no que se refere a esses temas. Portanto, tópicos representativos sobre conceitos e definições, objetivos, componentes e sistemas de BPM e de workflow, identificados na pesquisa bibliográfica e sintetizados no referencial teórico deste artigo, foram relacionados entre si para identificar complementaridades e distinções entre os temas investigados.

O referencial bibliográfico utilizado para a análise foi levantado a partir de pesquisa em bases de dados online como portal de periódicos CAPES e base Elsevier, por meio dos buscadores "Gerenciamento de Processos de Negócio", "Business Process Management", "Gestão / Gerenciamento de processos", "Workflow", e "Fluxo(s) de trabalho".

Entre as publicações brasileiras e internacionais obtidas por meio da aplicação desses buscadores nas bases de dados como as supracitadas, utilizou-se, como referências: artigos publicados nos periódicos Information Technology and Management, Crossroads, Business Process Management Jornal, Data \& Knowledge Engineering, livros de autoria de Weske, Verma, Miers, Jeston e Nelis (2006), Laudon e Laudon, Anais dos encontros Business Process Management International Conference e Wirtschaftsinformatik, e documentos publicados pelo Workflow Management CoalitioneGartnerGroup. Como referências nacionais, utilizou-se artigos publicados nos periódicos Revista de Informática Aplicada, RAE Eletrônica e Perspectivas em Ciência da Informação, livros de autoria de Baldam et al. (2007), Cruz (2006; 2010), Davenport (1994), De Sordi e Marinho (2005), artigos publicados nos Anais dos eventos Encontro Nacional de Engenharia de Produção, Simpósio de Administração Da Produção, Logística e Operações Internacionais e Encontro de Administração da Informação, e dissertações de Enoki e Costa, cujas contribuições à discussão sobre conceitos e práticas de BPM foram julgadas válidas para este estudo. 
Além de sua relevância para a identificação do estado-da-arte do BPM e workflow na literatura, e proposta de seu delineamento conceitual, essas obras foram selecionadas devido à sua inserção no período temporal considerado para a pesquisa: entre os anos 2000 e 2012, considerando-se exceções de referências relevantes publicadas em períodos anteriores, como no caso da obra de Davenport e documentos do Workflow Management Coalition, respectivamente publicados em 1994 e 1995. Esse período foi selecionado por constituir a época em que o BPM foi apresentado às comunidades científicas e de negócios, desenvolvido por ambas.

Desse referencial, extraíram-se conceitos, definições, componentes, áreas de atribuição, objetivos e menções a sistemas para operacionalização do BPM e do workflow. Tais conteúdos permitiram a síntese do estado da arte dos temas investigados. Em seguida, os conteúdos selecionados foram sintetizados na forma de tópicos, permitindo a identificação de sobreposições e elementos distintivos entre ambos os temas, apontados nas referências do estudo. Esses aspectos foram analisados à luz dos questionamentos quanto a complementaridades e limites existentes entre os conceitos de BPM e de workflow, originando quadros de correlação dos elementos teóricos da literatura e pertinentes a um ou outro conceito.

Por meio dos quadros foi possível esclarecer, enfim, limites teóricos que envolvem os temas investigados e desenvolver um modelo de delineamento conceitual entre BPM e workflow que viabiliza o correto uso de uma ou de outra denominação tanto no âmbito acadêmico, quanto organizacional.

\section{ESTADO DA ARTE E DISCUSSÃO DE RELAÇÕES ENTRE BPM E WORKFLOW}

A aplicação dos procedimentos metodológicos apresentados permitiu observar que as publicações nacionais de referência sobre BPM e sobre workflow privilegiam a abordagem de ferramentas, sistemas e padrões de operacionalização desses conceitos. Discussões de ordem conceitual ocorrem em segundo plano, seguidas por aquelas de ordem metodológica. Esse fato sugere que, nacionalmente, a preocupação com a implementação do BPM e do workflow prevalece apesar da multiplicidade de conceitos de ambos os temas, o que pode comprometer a própria implementação desses, se baseada em princípios equivocados.

O contrário ocorre nas publicações internacionais, que privilegiam o conceito de BPM e de workflow, discutindo como segundo principal enfoque, métodos, aplicações e gestão de ambas as temáticas. Nesse sentido entende-se que, internacionalmente, existe a preocupação em delinear conceitos e métodos desses temas antes de discutir sua inserção nos ambientes de negócio, o que teoricamente garante integridade e qualidade a este último processo. 
Comparativos ou pesquisas voltadas ao esclarecimento conceitual de ambos os temas, que poderiam ser úteis aos gestores de ambientes de negócio quando da seleção e implementação de modelos e tecnologias de gerenciamento de seus processos, ocorrem com menor frequência em ambas as categorias de publicações. Esse fato sugere que a preocupação em delimitar conceitos de BPM e de workflow tem menor urgência por parte da literatura, frente ao desenvolvimento de novos conceitos, à definição de métodos e à estruturação de tecnologias para operacionalização desses mecanismos.

Acredita-se que o mercado de desenvolvimento de Sistemas para aplicação do BPM e do workflow influencia em parte na consolidação do cenário de indefinição que os envolve e, no Brasil, influencia a prioridade atribuída à discussão de tecnologias em relação às definições e métodos. Essa crença é justificada pelo posicionamento estratégico dos próprios sistemas nos ambientes organizacionais como mecanismos de gestão de recursos, objetivos e mercados, e pela urgência de tais ambientes em adquirir e manter competitividade em espaços de atuação cada vez mais globalizados e cujas movimentações devem ser cada vez mais agilizadas.

As complementaridades e os limites teóricos existentes entre os conceitos de BPM e de workflow, seus objetivos, modelos de ciclo de vida, áreas de pertinência, componentes e tecnologias, identificadas durante a pesquisa, são sintetizadas nos quadros a seguir. Por meio deles é possível perceber que, embora a literatura retrate uma dificuldade generalizada em estabelecer definições únicas e mutuamente exclusivas para o Gerenciamento de Processos de Negócio e o workflow, ela própria possibilita a extração de elementos delimitadores de cada tema e a identificação de seus aspectos de sobreposição. O Quadro 1 sumariza, assim, conceitos e funções dos temas investigados na pesquisa, apontados pela literatura:

Quadro 1: Conceitos e funções de BPM e de workflow

\begin{tabular}{|c|c|c|c|}
\hline Tópico & Fonte & BPM & Workflow \\
\hline Disciplina & McCoy et al. (2007) & & \\
\hline Transdisciplinar & Ko (2009) & & \\
\hline \multirow[t]{2}{*}{ Tecnologia } & Costa (2009) & & \\
\hline & Verma (2009) & & \\
\hline $\begin{array}{l}\text { Ferramenta ou conjunto de } \\
\text { ferramentas de tecnologia }\end{array}$ & McCoy et al. (2007) & & \\
\hline $\begin{array}{l}\text { Conhecimentos ferramentais e } \\
\text { organizacionais }\end{array}$ & Cruz (2006) & & \\
\hline $\begin{array}{l}\text { Extensão, evolução ou sequência do } \\
\text { workflow }\end{array}$ & Ko (2009) & & \\
\hline Gerenciamento de fluxo de trabalho & Leite, Rezende (2007) & & \\
\hline
\end{tabular}




\begin{tabular}{|c|c|c|c|}
\hline Tópico & Fonte & BPM & Workflow \\
\hline Planejamento e gestão de processos & Amaral et al. (2008) & & \\
\hline $\begin{array}{l}\text { - Gerenciamento do ciclo de vida de } \\
\text { processos (operacionais) }\end{array}$ & Costa (2009) & & \\
\hline - Práticas gerenciais estruturadas & McCoy et al. (2007) & & \\
\hline \multirow[t]{2}{*}{-- Definição de processos } & Costa (2009) & & \\
\hline & $\begin{array}{l}\text { Weske, van der Aalst, } \\
\text { Verbeek (2004) }\end{array}$ & & \\
\hline \multirow[t]{3}{*}{-- Design de processos } & Amaral et al. (2008) & & \\
\hline & $\begin{array}{l}\text { Weske, van der Aalst, } \\
\text { Verbeek (2004) }\end{array}$ & & \\
\hline & Verma (2009) & & \\
\hline \multirow[t]{2}{*}{--- Modelagem de processos } & Amaral et al. (2008) & & \\
\hline & Verma (2009) & & \\
\hline \multirow[t]{5}{*}{-- Execução de processos } & Amaral et al. (2008) & & \\
\hline & $\begin{array}{l}\text { Weske, van der Aalst, } \\
\text { Verbeek (2004) }\end{array}$ & & \\
\hline & Verma (2009) & & \\
\hline & $\begin{array}{l}\text { De Sordie Marinho } \\
\text { (2005) }\end{array}$ & & \\
\hline & Cruz (2006) & & \\
\hline -- Representação visual dos processos & Leite, Rezende (2007) & & \\
\hline \multirow[t]{3}{*}{-- Controle de processos } & Amaralet al. (2008) & & \\
\hline & Baldamet al. (2007) & & \\
\hline & $\begin{array}{l}\text { Weske, van der Aalst, } \\
\text { Verbeek (2004) }\end{array}$ & & \\
\hline \multirow[t]{4}{*}{--- Monitoramento de processos } & Amaral et al. (2008) & & \\
\hline & $\begin{array}{l}\text { Weske, van der Aalst, } \\
\text { Verbeek (2004) }\end{array}$ & & \\
\hline & Baldamet al. (2007) & & \\
\hline & Verma (2009) & & \\
\hline \multirow[t]{3}{*}{--- Análise de processos } & Amaral et al. (2008) & & \\
\hline & $\begin{array}{l}\text { Weske, van der Aalst, } \\
\text { Verbeek (2004) }\end{array}$ & & \\
\hline & Verma (2009) & & \\
\hline --- Simulação de processos & & & \\
\hline
\end{tabular}




\begin{tabular}{|c|c|c|c|}
\hline Tópico & Fonte & BPM & Workflow \\
\hline Coordenação de processos & \multirow[t]{2}{*}{ Costa (2009) } & & \\
\hline \multirow[t]{2}{*}{ Automação de fluxo de trabalho } & & & \\
\hline & $\begin{array}{l}\text { De Sordi e Marinho } \\
\text { (2005) }\end{array}$ & & \\
\hline \multirow[t]{3}{*}{ Automação de processos } & Leite, Rezende (2007) & & \\
\hline & Costa (2009) & & \\
\hline & $\begin{array}{l}\text { Montaniniet al. } \\
(2008)\end{array}$ & & \\
\hline \multirow[t]{2}{*}{ - Automatização de processos } & Amaral et al. (2008) & & \\
\hline & Verma (2009) & & \\
\hline $\begin{array}{l}\text { - Gerenciamento holístico de } \\
\text { processos }\end{array}$ & & & \\
\hline Ações resultantes de eventos & & & \\
\hline
\end{tabular}

Fonte: Da autora (2012)

Diante do conteúdo do Quadro 1, observou-se que os conceitos de BPM e de workflow se sobrepõem ou igualam no sentido de que ambos constituem disciplinas, tecnologias ou ferramentas, e atuam na automação integral ou parcial de processos e no ciclo de vida de processos de negócio. Este último fator permite diferenciar ambos os conceitos, pois somente o BPM atua no planejamento e na gestão estruturada e holística de processos, integrando stakeholders do negócio, ao passo que o workflow atua de maneira menos expressiva nas tarefas de: definição, design, modelagem, execução, representação, monitoramento e análise de processos mantendo foco no fluxo de trabalho neles embutido e automatizado.

Portanto, ao passo que o BPM abrange questões culturais e de integração entre componentes de um negócio, compreendendo seu contexto gerencial mais abrangente, o workflow consiste no fluxo de trabalho inserido nos componentes menores desse contexto, estando inserido no âmbito pragmático de regras e estruturas de trabalho simplificadas, que movem processos do ambiente em questão. Essa delimitação entre ambos os conceitos é perceptível também nos objetivos de cada qual, propostos pela literatura e resumidos no Quadro 2: 
Quadro 2: Objetivos do BPM e do workflow

\begin{tabular}{|c|c|c|c|}
\hline Tópico & Fonte & BPM & Workflow \\
\hline \multirow{5}{*}{$\begin{array}{l}\text { Viabilizar trâmite de } \\
\text { documentos }\end{array}$} & McCoy et al. (2007) & & \\
\hline & Ko (2009) & & \\
\hline & Laudon, Laudon (2007) & & \\
\hline & $\begin{array}{l}\text { Workflow Management } \\
\text { Coalition (1995) }\end{array}$ & & \\
\hline & Verma (2009) & & \\
\hline - Eliminar trânsito de papel & \multirow[t]{2}{*}{ Ko (2009) } & & \\
\hline $\begin{array}{l}\text { Viabilizar transações de um } \\
\text { negócio }\end{array}$ & & & \\
\hline \multirow[t]{2}{*}{ Passar tarefas entre pessoas } & Verma (2009) & & \\
\hline & $\begin{array}{l}\text { Workflow Management } \\
\text { Coalition (1995) }\end{array}$ & & \\
\hline $\begin{array}{l}\text { Transmitir informações por } \\
\text { rotinas }\end{array}$ & \multirow[t]{2}{*}{ Ko (2009) } & & \\
\hline Simplificar procedimentos & & & \\
\hline \multirow[t]{3}{*}{ - Automatizar procedimentos } & Laudon, Laudon (2007) & & \\
\hline & $\begin{array}{l}\text { Workflow Management } \\
\text { Coalition (1995) }\end{array}$ & & \\
\hline & Cruz (2006) & & \\
\hline $\begin{array}{l}\text { Modificar processos em tempo } \\
\text { real }\end{array}$ & Verma (2009) & & \\
\hline \multirow{5}{*}{$\begin{array}{l}\text { Monitorar / avaliar / estabilizar } \\
\text { desempenho de processos }\end{array}$} & Montaniniet al. (2008) & & \\
\hline & Cruz (2010) & & \\
\hline & Reijers, Song, Jeong (2007) & & \\
\hline & Miers (2006) & & \\
\hline & Amaral et al. (2008) & & \\
\hline \multirow[t]{2}{*}{$\begin{array}{l}\text { - Atribuir eficiência e } \\
\text { efetividade a processos }\end{array}$} & $\begin{array}{l}\text { Weske, van der Aalst, } \\
\text { Verbeek (2004) }\end{array}$ & & \\
\hline & Laudon, Laudon (2007) & & \\
\hline \multirow{4}{*}{$\begin{array}{l}\text { Integrar fatores humano e } \\
\text { tecnológicos dos processos }\end{array}$} & Leite, Rezende (2007) & & \\
\hline & Costa (2009) & & \\
\hline & Enoki (2006) & & \\
\hline & Verma (2009) & & \\
\hline
\end{tabular}

Fonte: Da autora (2012) 
A partir do Quadro 2, nota-se que o workflow tem foco principalmente na etapa de execução de processos inserida no ciclo de vida do BPM, que viabiliza o trâmite de documentos entre atividades e a realização de transações do negócio, a eliminação do uso de papel na realização do trabalho, sua simplificação e inserção em rotinas que atribuem eficiência e efetividade aos processos. O conteúdo do Quadro 2 reforça a pertinência do BPM à integração entre stakeholders de um negócio, e deles com tecnologias utilizadas no ciclo de vida de processos. O Quadro reforça, também, a ocupação do BPM com o desempenho dos processos que, entende-se, é auxiliada pelo workflow.

Diante das definições baseadas nos Quadros 1 e 2, o workflow aparenta ter menor valor em relação ao Gerenciamento de Processos de Negócio para o gerenciamento íntegro e integrado do mesmo negócio. As vantagens do workflow, entretanto, são apontadas por Cruz (2010): ser estruturado sobre um modelo conceitual universalmente aceito, que viabiliza a comparação entre soluções de sistemas para operacionaliza-lo, e ser gerido por um órgão regulador que se encarrega de manter a padronização das práticas em sua área.

O caráter de disciplina de ambos os conceitos é traduzido nas áreas às quais são pertinentes, além da gestão de processos e da gestão de fluxos de trabalho propriamente ditas. Já os modelos conceituais e ciclos de vida pressupõem componentes do BPM e dos fluxos de trabalho, enquanto conceitos e plataformas operacionalizadas por sistemas. Em relação a esses componentes, a literatura analisada demonstra certa unicidade, conforme o conteúdo do Quadro 3:

Quadro 3: Componentes do BPM e do workflow

\begin{tabular}{|c|c|c|c|}
\hline Tópico & Fonte & BPM & Workflow \\
\hline \multirow[t]{3}{*}{ Métodos } & Cruz (2006) & & \\
\hline & McCoy et al. (2007) & & \\
\hline & Weske, van der Aalst, Verbeek & & \\
\hline \multirow[t]{5}{*}{ Políticas e Regras } & $(2004)$ & & \\
\hline & Enoki (2006) & & \\
\hline & Costa (2009) & & \\
\hline & $\begin{array}{l}\text { Workflow Management } \\
\text { Coalition (1995) }\end{array}$ & & \\
\hline & McCoy et al. (2007) & & \\
\hline \multirow[t]{3}{*}{ Métricas } & & & \\
\hline & Cruz (2010) & & \\
\hline & Montaniniet al. (2008) & & \\
\hline \multirow{2}{*}{ Práticas } & Verma (2009) & & \\
\hline & Cruz (2010) & & \\
\hline
\end{tabular}




\begin{tabular}{|c|c|c|c|}
\hline Tópico & Fonte & BPM & Workflow \\
\hline \multirow[t]{2}{*}{ Técnicas } & $\begin{array}{l}\text { Weske, van der Aalst, Verbeek } \\
\text { (2004) }\end{array}$ & & \\
\hline & Costa (2009) & & \\
\hline \multirow{3}{*}{$\begin{array}{l}\text { Ferramentas de Tecnologia } \\
\text { da Informação }\end{array}$} & McCoy et al. (2007) & & \\
\hline & Cruz (2006) & & \\
\hline & $\begin{array}{l}\text { Weske, van der Aalst, Verbeek } \\
\text { (2004) }\end{array}$ & & \\
\hline \multirow[t]{2}{*}{ Papéis e responsabilidades } & Weske (2007) & & \\
\hline & Ko (2009) & & \\
\hline \multirow[t]{2}{*}{ Tarefas } & Costa (2009) & & \\
\hline & Workflow Management & & \\
\hline \multirow[t]{3}{*}{ Modelos } & Coalition (1995) & & \\
\hline & Costa (2009) & & \\
\hline & $\begin{array}{l}\text { Weske, van der Aalst, Verbeek } \\
\text { (2004) }\end{array}$ & & \\
\hline
\end{tabular}

Fonte: Da autora (2012)

O conteúdo do Quadro 3 aponta que somente métodos, neste caso relativos à gestão de processos de negócio, são exclusividade do BPM. Modelos, ferramentas de Tecnologia da Informação, técnicas, métricas, políticas, regras, papéis e responsabilidades são comuns ao BPM e ao workflow. A realização de tarefas é apresentada como único componente específico desse último conceito, embora quando confrontada com a execução do ciclo de vida de processos, seja também pertinente a ambos os temas tratados nesta pesquisa.

Após a sistematização de conceitos, objetivos, componentes e áreas de pertinência do workflow e do BPM, foram reunidas da literatura definições e componentes dos WfMS e dos BPMS (Quadro 4). 
Quadro 4: Definições e componentes de BPMS e de WfMS

\begin{tabular}{|c|c|c|c|}
\hline Tópico & Fonte & BPMS & WfMS \\
\hline Suporte a processos de negócio & Dubouloz (2004 apud OLIVEIRA et al., 2010) & & \\
\hline \multirow{4}{*}{$\begin{array}{l}\text { Melhoria de (eficiência de) } \\
\text { processos }\end{array}$} & McCoy et al. (2007) & & \\
\hline & Ko (2009) & & \\
\hline & Cruz (2006) & & \\
\hline & Reijers, Song, Jeong (2007) & & \\
\hline \multirow{2}{*}{$\begin{array}{l}\text { Suporte ao ciclo de vida do } \\
\text { processo de negócio }\end{array}$} & Leite, Rezende (2007) & & \\
\hline & $\begin{array}{l}\text { Business Process Management Initiative } \\
\text { apud Cruz (2010) }\end{array}$ & & \\
\hline \multirow[t]{2}{*}{ Desenho de processos } & Cardoso, Bostrom, Sheth (2004) & & \\
\hline & Enoki (2006) & & \\
\hline \multirow[t]{2}{*}{ - Design de processos } & Verma (2009) & & \\
\hline & Ko, Lee, Lee (2009) & & \\
\hline \multirow[t]{2}{*}{ - Modelagem de processos } & Leite, Rezende (2007) & & \\
\hline & Verma (2009) & & \\
\hline - Representação de processos & Weske, van der Aalst, Verbeek (2004) & & \\
\hline $\begin{array}{l}\text { Registro momentâneo de } \\
\text { processos }\end{array}$ & Cardoso, Bostrom, Sheth (2004) & & \\
\hline \multirow{2}{*}{$\begin{array}{l}\text { Monitoramento e análise de } \\
\text { processos }\end{array}$} & Enoki (2006) & & \\
\hline & Costa (2009) & & \\
\hline \multicolumn{4}{|l|}{$\begin{array}{l}\text { - Acompanhamento de } \\
\text { desempenho de processos }\end{array}$} \\
\hline - Diagnóstico de processos & Leite, Rezende (2007) & & \\
\hline Integração entre processos & Dubouloz (2004 apud OLIVEIRA et al., 2010) & & \\
\hline \multirow[t]{2}{*}{ Gestão de processos } & Cardoso, Bostrom, Sheth (2004) & & \\
\hline & Amaralet al. (2008) & & \\
\hline \multirow[t]{2}{*}{ Execução de processos de negócio } & $\begin{array}{l}\text { Business Process Management Initiative } \\
\text { apud Cruz (2010) }\end{array}$ & & \\
\hline & Amaral et al. (2008) & & \\
\hline \multirow{5}{*}{$\begin{array}{l}\text { Gerenciamento e } \\
\text { operacionalização do BPM } \\
\text { simultaneamente }\end{array}$} & Cruz (2006) & & \\
\hline & Reijers, Song, Jeong (2007) & & \\
\hline & Weske, van der Aalst, Verbeek (2004) & & \\
\hline & Miers (2006) & & \\
\hline & Enoki (2006) & & \\
\hline
\end{tabular}




\begin{tabular}{|c|c|c|c|}
\hline Tópico & Fonte & BPMS & WfMS \\
\hline Automatização de organizações & Cruz (2010) & & \\
\hline \multirow{3}{*}{$\begin{array}{l}\text { - Automatização de processos e } \\
\text { procedimentos }\end{array}$} & Ko (2009) & & \\
\hline & Laudon, Laudon (2007) & & \\
\hline & Cruz (2010) & & \\
\hline Transferência de dados & Dubouloz (2004 apud OLIVEIRA et al., 2010) & & \\
\hline \multirow[t]{4}{*}{ Automação de fluxo de trabalho } & Weske, van der Aalst, Verbeek (2004) & & \\
\hline & Workflow Management Coalition (1995) & & \\
\hline & Weske (2007) & & \\
\hline & \multirow[t]{2}{*}{ De Sordi e Marinho (2005) } & & \\
\hline $\begin{array}{l}\text { - Definição, criação e execução de } \\
\text { fluxos de trabalho }\end{array}$ & & & \\
\hline \multirow{3}{*}{$\begin{array}{l}\text { Conexão e interação com outros } \\
\text { softwares }\end{array}$} & Weske (2007) & & \\
\hline & Enoki (2006) & & \\
\hline & $\begin{array}{l}\text { Business Process Management Initiative } \\
\text { apud Cruz (2010) }\end{array}$ & & \\
\hline \multirow{5}{*}{$\begin{array}{l}\text { Integração entre stakeholders de } \\
\text { um negócio }\end{array}$} & Cruz (2010) & & \\
\hline & Reijers, Song, Jeong (2007) & & \\
\hline & Cruz (2006) & & \\
\hline & Amaral et al. (2008) & & \\
\hline & Montaniniet al. (2008) & & \\
\hline $\begin{array}{l}\text { - Integração entre pessoas e } \\
\text { tecnologias }\end{array}$ & Verma (2009) & & \\
\hline - Colaboração & Leite, Rezende (2007) & & \\
\hline Independente e interoperável & Montaniniet al. (2008) & & \\
\hline \multirow[t]{2}{*}{ Novo formato para WfMS } & Weske (2007) & & \\
\hline & \multirow[t]{2}{*}{ Cruz (2010) } & & \\
\hline Funcionalidade do BPMS & & & \\
\hline
\end{tabular}

Fonte: Da Autora (2012)

O conteúdo do Quadro 4 reforça definições já estabelecidas para o BPM e o workflow, como o papel exclusivo do segundo no que tange a definir, executar, controlar e automatizar fluxos trabalho, bem como a atuação conjunta de ambos no que se refere à obtenção de eficiência por parte do negócio em que estão inseridos.

O suporte ao ciclo de vida de processos de negócio, apontado no Quadro 4 como definição única dos BPMS, se alinha à definição constante no Quadro 1, para o BPM, em relação ao mesmo ciclo. O workflow, assim, oferece suporte (Quadro 1) e funcionalidades (Quadro 4) para a execução de tarefas do ciclo de vida de processos, entre as quais constam o desenho, o registro e a modelagem, entretanto, não atua na concepção de todo o conjunto de tarefas que compreende o Gerenciamento de Processos de Negócio. Essa constatação é exemplificada pelas tarefas inseridas na etapa de avaliação, no ciclo de vida desses processos.

Por conseguinte, ocorre a atribuição aos BPMS, e não aos WfMS, a função de gerir e operacionalizar simultaneamente o BPM. O papel de facilitar a interação entre stakeholders e tecnologias, inserido no Quadro 4 como viabilizado por BPMS e por WfMS, é apresentado de forma idêntica no Quadro 2 e no Quadro 3, respectivamente em 
relação aos objetivos do BPM e do workflow e aos papéis e responsabilidades de ambos. Já no Quadro 1, a citada integração é relacionada como conceito do BPM, apenas.

\subsection{Delineamento conceitual do bpm e do workflow}

As complementaridades existentes entre os conceitos de BPM e de workflow, que levam ao seu tratamento como elementos similares e até sobrepostos, foram identificadas por meio da análise de publicações referentes a ambos, da última década. O mesmo processo, resultante nos Quadros já apresentados neste artigo, possibilitou a identificação das diferenças entre ambos os conceitos investigados, que os delimitam quanto a objetivos, áreas, componentes, funções e sistemas, e permitem identificar a forma correta de utilizar uma ou outra denominação.

Tais definições permitem traçar o delineamento conceitual do BPM e do workflow, sintetizado na Figura 2. A área central da Figura demonstra os aspectos de sobreposição do BPM e do workflowe destaca, em preto, os aspectos que se referem aos Sistemas que operacionalizam cada qual, em seu respectivo círculo.

Figura 2: Modelo de delineamento conceitual entre BPM e workflow

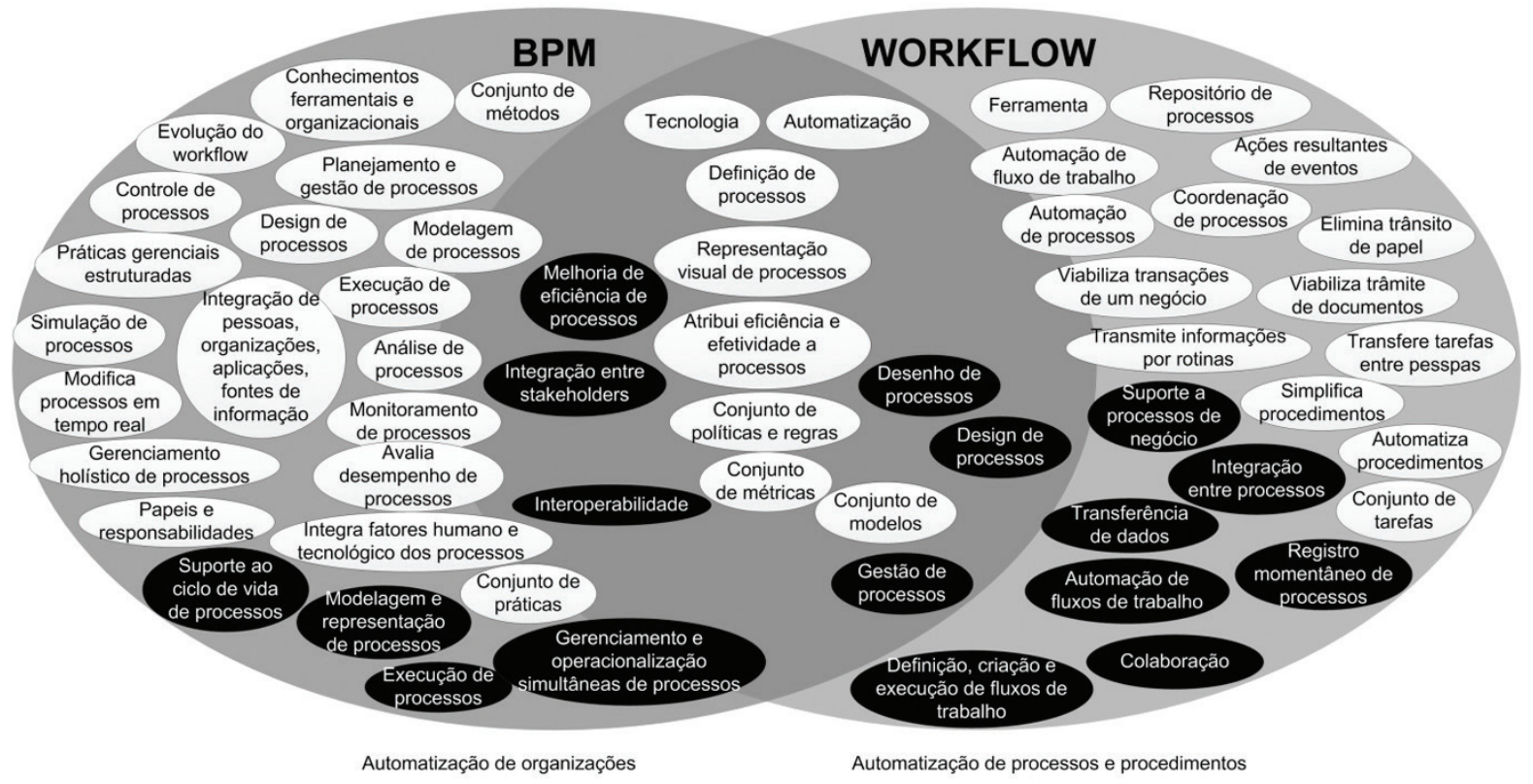

Fonte: Da autora (2012)

A partir da Figura 2 percebe-se a priorização, por parte do BPM, na automatização de organizações e na integração de componentes complexos, em maioria metodológicos. Nota-se que o workflow, por sua vez, é voltado à automatização de processos e procedimentos, característica que justifica seus componentes em maioria de ordem operacional. A interseção entre ambos contempla elementos do ciclo de vida de processos de negócio, corroborando a demonstração da Figura 1 na qual ambos os conceitos atuam no mesmo ciclo em tarefas distintas. A mesma interseção demonstra, enfim, 
que o Gerenciamento de Processos de Negócio e os fluxos de trabalho compartilham elementos inerentes a qualquer conceito relacionado à gestão organizacional, que se desdobra em aplicações, a saber: técnicas, métricas, modelos, regras e tecnologias.

De acordo com as definições sintetizadas nos Quadros 1 a 4 e com o modelo proposto, é possivel delimitar, enfim, que:

O BPM compreende a gestão e a coordenação do ciclo de vida de processos de negócio, stakeholders e tecnologias associadas, permitindo a realização do planejamento e do controle desse ciclo por meio de métodos e técnicas adaptáveis a cada organização e a sua respectiva cultura. Essas tarefas são operacionalizadas por meio de BPMS, que auxiliam na execução e na integração dos processos neles inseridos, corroborando o papel do BPM na integração de componentes de negócio, que visa à melhoria de seu desempenho, de maneira holística.

O workflow, por sua vez, representa os fluxos de trabalho, automatizados ou não, das operações organizacionais. Tais fluxos distribuem tarefas e informações entre seus executores (sistemas, pessoas ou ambos) por meio de regras de negócio explícitas, simplificadas e aderentes a um modelo de referência universalmente aceito e inserido nos WfMS. Esses sistemas de workflow oferecem suporte aos BPMS à medida que promovem a eficiência do trabalho e viabilizam o fluxo eletrônico de documentos em organizações.

\section{CONSIDERAÇÕES FINAIS}

A ausência de limites consolidados entre o BPM e o workflow, enquanto conceitos e no que tange a seus respectivos sistemas, é percebida analisando-se o conteúdo da literatura pertinente e, principalmente, o ambiente organizacional, em que os diversos modelos gerenciais já inseridos contribuem para uma mescla de atitudes que nem sempre beneficia as operações do negócio ou facilita seu gerenciamento. Conforme se observou durante esta pesquisa, tal indefinição deve permanecer até que a própria literatura e o próprio ambiente organizacional estabeleçam resoluções universalmente aceitas e distintas, passando a adotá-las.

Algumas entre essas definições já são propostas, de acordo com os comparativos realizados quanto a conceito, objetivo, áreas de pertinência, componentes e sistemas de BPM e de workflow. Nesse sentido, os resultados deste estudo representam um ponto de partida do esclarecimento conceitual de tais temas, contribuindo para o desenvolvimento das teorias e práticas que os envolvem e para a continuidade de estudos relacionados à proposta de limites de sua aplicabilidade.

As definições aqui propostas são baseadas em um número restrito de referências, e não necessariamente serão aplicáveis de forma plena na prática, pois esta pesquisa baseou-se 
exclusivamente em investigação teórica, não abrangendo a visão das organizações sobre quais poderiam ser conceitos delimitados de cada tema abordado. Uma investigação nesse sentido é recomendada como complemento aos resultados aqui demonstrados.

É pertinente ainda, a partir dos resultados desta discussão, a verificação da aderência da delimitação proposta no âmbito teórico, à visão identificada no âmbito prático, comparando ambas e integrando-as em um modelo unificado que caracterize o Gerenciamento de Processos de Negócio e os fluxos de trabalho.

\title{
MANAGING BUSINESS PROCESSES AND WORKFLOW: STATE OF THE ART DESIGN AND CONCEPTUAL OUTLINE
}

\begin{abstract}
Managing processes requires, by the actors in the context of business, the understanding of concepts, models and tools appropriate to that function. The Business Process Management (BPM) provides the managers of these environments the possibility of integrating people and technology to operationalize processes and achieve organizational objectives. BPM, treated as a set of methodologies and tools, comes from other mechanisms of management such as workflow. The similarity of designs and applications, as well as the historical relationship between workflows and processes, led to their treatment as synonyms concerning the content of literature and managed environments through these models. This form of treatment hinders the correct adoption of one or another denomination mechanisms and tools for organizational management in addition to compromise investigative processes in a scientific context. Definitions, components, applications and goals of BPM and workflow were analyzed through literature research, national and international, of material published between 2000 and 2012. Specificities and complementarities between both were raised in order to characterize each and define their conceptual overlap, culminating in analysis frameworks that allow the visualization of boundaries between them. It is finally shown a proposal for a conceptual design of the research topics, contemplating their individual and mutual characteristics, to enable the correct use of each term in the academic and technical contexts.
\end{abstract}

Keywords: Management of Business Processes. Workflow. Lifecycle of business processs. 


\section{REFERÊNCIAS}

AMARAL, F. P. et al. O papel das ferramentas para sistematização de processos de negócios (BPMS). In: ENCONTRO NACIONAL DE ENGENHARIA DE PRODUÇÃO, 28., 2008, Rio de Janeiro. Anais..., Rio de Janeiro: ABEPRO, 2008. Disponível em: <http:// www.abepro.org.br/biblioteca/enegep2008_TN_STO_076_536_11940.pdf>. Acesso em: 02 out. 2011.

BALDAM, R. et al. Gerenciamento de processos de negócios: BPM - business process management. 2. ed.São Paulo: Érica, 2007.

CARDOSO, J.; BOSTROM, R. P.; SHETH, A. Workflow management systems and ERP systems: differences, commonalities, and applications. Information Technology and Management, v. 5, n. 3-4, p. 319-338, 2004.Disponível em: <http://www.springerlink. com/index/x12g4806t3483220.pdf>. Acesso em: 02 out. 2011.

COSTA, L. Formulação de uma metodologia de modelagem de processos de negócio para implementação de workflow. 2009. 130 f. Dissertação (Mestrado em Engenharia de Produção) - Universidade Tecnológica Federal do Paraná, Ponta Grossa, 2009. Disponível em: <http://www.pg.utfpr.edu.br/dirppg/ppgep/dissertacoes/arquivos/112/ Dissertacao.pdf>. Acesso em: 02 out. 2011.

CRUZ, T. BPM \& BPMS. Rio de Janeiro: Brasport, 2010.

Uso e desuso de sistemas de workflow: porque as organizações não conseguem obter retorno, nem sucesso, com investimentos em projetos de workflow. Rio de Janeiro: E-papers Serviços Editoriais, 2006.

DAVENPORT, T. H. Reengenharia de processos: como inovar na empresa através da tecnologia da informação. Rio de Janeiro: Campus, 1994.

DE SORDI, J.O.; MARINHO, B. L. Conectividade entre organizações: análise do potencial dos ambientes especializados na integração entre sistemas de informação em colaborar com a gestão de operações. In: SIMPÓSIO DE ADMINISTRAÇÃO DA PRODUÇÃO, LOGÍSTICA E OPERAÇÕES INTERNACIONAIS - SIMPOI, 8., 2005, São Paulo. Anais..., São Paulo: FGV, 2005. v. 1. Disponível em: <http://www.simpoi.fgvsp.br/ arquivo/2005/artigos/E2005_T00039_PCN39204.pdf>. Acesso em: 02 out. 2011. 
ENOKI, C. Gestão de processos de negócio: uma contribuição para a avaliação de soluções de business process management (BPM) sob a ótica da estratégia de operações. 225 f. 2006. Dissertação (Mestrado em Engenharia) - Escola Politécnica, Universidade de São Paulo, São Paulo, 2006. Disponível em: <http://www.teses.usp.br/ teses/disponiveis/3/3136/tde-01122006-170526/pt-br.php>. Acessoem: 01 out. 2011.

JESTON, J.; NELIS, J. Business process management: practical guidelines for successful implementations. 1. ed. Oxford: Elsevier, 2006.

KO, R. K. L. A computer scientist's introductory guide to business process management (BPM).Crossroads, v. 15, n. 4, jun. 2009. Disponível em: <http://portal.acm.org/ citation.cfm?id=1558901>. Acesso em: 02 out. 2011.

KO, R. K. L.; LEE, S. S. G.; LEE, E. W. Business process management (BPM) standards: a survey. Business Process Management Journal, v. 15, n. 5, 2009, p. 744-791. Disponível em: <http://www.emeraldinsight.com/journals.htm?articleid=1811155\&show=abstra ct>. Acesso em: 05 out. 2011.

LAUDON, K. C., LAUDON, J. P. Sistemas de informação gerenciais. 7. ed. São Paulo: Pearson Prentice Hall, 2007.

LEITE, L. O.; REZENDE, D. A. Gestão corporativa por processos na administração pública municipal: estudo de caso da implantação de business process management (BPM) no Instituto Curitiba de Informática. In: ENCONTRO DE ADMINISTRAÇÃO DA INFORMAÇÃO, 1., 2007, Florianópolis. Anais..., Florianópolis: ENADI, 2007. Disponível em: <http://www.anpad.org.br/evento.php?acao=subsecao\&cod_edicao_ subsecao=292\&interna=true\&cod_evento_edicao=34>. Acesso em: 13 out. 2011.

MCCOY, D. W. et al. Key issues for business process management. GartnerGroup, 2007. Disponível em: <http://www.wfmc.org/Download-document/Gartner-Key-IssuesResearch-Notes-on-BPM.html>. Acesso em: 25 jul. 2011.

MIERS, D. The key issues to BPM project success. In: FISCHER, L. (Ed.) Workflow handbook. Lighthouse Point: Future Strategies, 2006.

MONTANINI, A. et al. BPM: gerenciando processos de negócios e impactos humanos. Revista de Informática Aplicada, v. 4, n. 1, jan./jun. 2008. Disponível em: <http://seer. uscs.edu.br/index.php/revista_informatica_aplicada/article/viewArticle/298>. Acesso em: 02 out. 2011. 
MORENO, V.; SANTOS, L. H. A. dos. Gestão do conhecimento e redesenho de processos de negócio: proposta de uma metodologia integrada, Perspectivas em Ciência da Informação, v.17, n.1, p.203-230, jan./mar. 2012. Disponível em: <http:// portaldeperiodicos.eci.ufmg.br/index.php/pci/article/view/1281>. Acesso em: 15 maio 2012.

OLIVEIRA, A. M. A. de et al. Avaliação de ferramentas de Business Process Management (BPMS) pela ótica da gestão do conhecimento. Perspectivas em Ciência da Informação, v. 15, n. 1, abr. 2010. Disponível em: <http://www.scielo.br/scielo.php?pid=S141399362010000100008\&script=sci_arttext\&tIng=es>. Acesso em: 02 out. 2011.

PÁDUA, S. I. D. de.; BISPO, C. A. F. Sistema de gerenciamento de workflow: um overview e um estudo de caso. In: ENCONTRO NACIONAL DE ENGENHARIA DE PRODUÇÃO, 23., 2003, Ouro Preto. Anais...,Ouro Preto: UFMG, 2003.

RAUTENBERG, S.; TODESCO, J. L.; STEIAL, A. V. Ontologias de domínio no mapeamento de instrumentos da gestão do conhecimento e de agentes computacionais da engenharia do conhecimento: o estado da arte, Perspectivas em Ciência da Informação, v.15, n.2, p.163-182, maio/ago. 2010. Disponível em: <http:// portaldeperiodicos.eci.ufmg.br/index.php/pci/article/view/826/739>. Acesso em: 15 out. 2011.

REIJERS, H. A.; SONG, M.; JEONG, B. On the performance of workflow processes with distributed actors: does place matter? In: ALONSO, G.; DADAM, P.; ROSEMANN, M. (Eds.) BUSINESS PROCESS MANAGEMENT INTERNATIONAL CONFERENCE, 5., 2007, Brisbane, Australia, set. 2007, Proceedings.... Brisbane: Springer, 2007. p. 32-47. Disponível em: <http://www.springerlink.com/content/g346404731m2/\#section=3723 69\&page $=5 \&$ locus=31>. Acesso em: 01 out. 2011.

SANTOS, L. R. Gestão da maturidade de processos essenciais -convergência para o futuro, RAE Eletrônica, São Paulo, v. 2, n. 1, jan./jun.,p. 1-14, 2003. Disponível em:<http://redalyc.uaemex.mx/src/inicio/ArtPdfRed.jsp?iCve $=205115557002>$. Acesso em: 11 out. 2011.

VERMA, N. Business process management: profiting from process. Nova Delhi: Global IndiaPublications PVT, 2009.

WESKE, M. Business process management: concepts, languages, architectures. Berlin: Springer, 2007. 
WESKE, M.; VAN DER AALST, W. M. P.; VERBEEK, H. M. W. Advances in business process management.Data \&Knowledge Engineering, v. 50, p. 1-8, 2004.Disponível em: $<$ http://citeseerx.ist.psu.edu/viewdoc/download?doi=10.1.1.83.8666\&rep=rep1\&type= pdf>. Acesso em: 02 out. 2011.

WORKFLOW MANAGEMENT COALITION. The workflow reference model, Hampshire, i. 1.1, jan. 1995. Disponível em: <http://www.wfmc.org/index.php?option=com_ docman\&task=doc_download\&gid=92\&ltemid=72>. Acesso em: 01 out. 2011.

\section{SOBRE A AUTORA}

Mestre em Ciência, Gestão e Tecnologia da Informação (UFPR), bacharel
em Gestão da Informação (UFPR). Desenvolve pesquisas em Fluxos de
Informação, mais especificamente sobre a avaliação e gestão desses
por meio dos princípios do Lean Thinking (Mentalidade Enxuta).
Autora do conceito de Fluxo Enxuto de Informação, atuando em sua
implementação atrelada ao Lean Office (Escritório Enxuto) em ambientes
de escritório. Estuda Sistemas de Gerenciamento de Processos de Negócio,
desenvolvendo instrumento para análise desses Sistemas com base
em domínios metodológico e ferramental do BPM. Tem experiência em
projetos nas áreas de: Gestão de Fluxos de Informação e Lean Office; Estudo
e resolução de problemas de informação em ambientes de escritório;
Educação continuada, Educação corporativa e Educação à Distância.

